Supporting Information

\title{
Density Functional Theory Study of the Decarboxylation and Decarbonylation of Acetic Acid on Pd (111)
}

Kingsley C. Chukwu and Líney Árnadóttir*

School of Chemical, Biological and Environmental Engineering, Oregon State University,

Corvallis, OR, 97331, United States.

Scheme S1. DCX pathways investigated in acetic acid decomposition over Pd (111).

DCX 1: $\mathrm{CH}_{3} \mathrm{COOH} \rightarrow \mathrm{CH}_{3} \mathrm{COO}+\mathrm{H} \rightarrow \mathrm{CO}_{2}+\mathrm{H}+\mathrm{CH}_{3}$

DCX 2: $\mathrm{CH}_{3} \mathrm{COOH} \rightarrow \mathrm{CH}_{3} \mathrm{COO}+\mathrm{H} \rightarrow \mathrm{CH}_{2} \mathrm{COO}+2 \mathrm{H} \rightarrow \mathrm{CO}_{2}+\mathrm{CH}_{2}+2 \mathrm{H}$

DCX 3: $\mathrm{CH}_{3} \mathrm{COOH} \rightarrow \mathrm{CH}_{3} \mathrm{COO}+\mathrm{H} \rightarrow \mathrm{CH}_{2} \mathrm{COO}+2 \mathrm{H} \rightarrow \mathrm{CHCOO}+3 \mathrm{H} \rightarrow \mathrm{CO}_{2}+3 \mathrm{H}+\mathrm{CH}$

DCX 4: $\mathrm{CH}_{3} \mathrm{COOH} \rightarrow \mathrm{CH}_{3} \mathrm{COO}+\mathrm{H} \rightarrow \mathrm{CH}_{2} \mathrm{COO}+2 \mathrm{H} \rightarrow \mathrm{CHCOO}+3 \mathrm{H} \rightarrow \mathrm{CCOO}+4 \mathrm{H} \rightarrow \mathrm{CO}_{2}$ $+4 \mathrm{H}+\mathrm{C}$

DCX 5: $\mathrm{CH}_{3} \mathrm{COOH} \rightarrow \mathrm{CH}_{3}+\mathrm{COOH} \rightarrow \mathrm{CH}_{3}+\mathrm{CO}_{2}+\mathrm{H}$

DCX 6: $\mathrm{CH}_{3} \mathrm{COOH} \rightarrow \mathrm{CH}_{2} \mathrm{COOH}+\mathrm{H} \rightarrow \mathrm{COOH}+\mathrm{CH}_{2}+\mathrm{H} \rightarrow \mathrm{CO}_{2}+\mathrm{CH}_{2}+2 \mathrm{H}$

DCX 7: $\mathrm{CH}_{3} \mathrm{COOH} \rightarrow \mathrm{CH}_{2} \mathrm{COOH}+\mathrm{H} \rightarrow \mathrm{CHCOOH}+2 \mathrm{H} \rightarrow \mathrm{COOH}+2 \mathrm{H}+\mathrm{CH} \rightarrow \mathrm{CO}_{2}+3 \mathrm{H}+$ $\mathrm{CH}$

$\mathrm{DCX} 8: \mathrm{CH}_{3} \mathrm{COOH} \rightarrow \mathrm{CH}_{2} \mathrm{COOH}+\mathrm{H} \rightarrow \mathrm{CHCOOH}+2 \mathrm{H} \rightarrow \mathrm{CCOOH}+3 \mathrm{H} \rightarrow \mathrm{COOH}+3 \mathrm{H}+\mathrm{C}$ $\rightarrow \mathrm{CO}_{2}+4 \mathrm{H}+\mathrm{C}$

DCX 9: $\mathrm{CH}_{3} \mathrm{COOH} \rightarrow \mathrm{CH}_{2} \mathrm{COOH}+\mathrm{H} \rightarrow \mathrm{CH}_{2} \mathrm{COO}+2 \mathrm{H} \rightarrow \mathrm{CHCOO}+3 \mathrm{H} \rightarrow \mathrm{CO}_{2}+3 \mathrm{H}+\mathrm{CH}$

DCX 10: $\mathrm{CH}_{3} \mathrm{COOH} \rightarrow \mathrm{CH}_{2} \mathrm{COOH}+\mathrm{H} \rightarrow \mathrm{CHCOOH}+2 \mathrm{H} \rightarrow \mathrm{CHCOO}+3 \mathrm{H} \rightarrow \mathrm{CO}_{2}+3 \mathrm{H}+\mathrm{CH}$

$\mathrm{DCX}$ 11: $\mathrm{CH}_{3} \mathrm{COOH} \rightarrow \mathrm{CH}_{2} \mathrm{COOH}+\mathrm{H} \rightarrow \mathrm{CHCOOH}+2 \mathrm{H} \rightarrow \mathrm{CCOOH}+3 \mathrm{H} \rightarrow \mathrm{CCOO}+4 \mathrm{H}$ $\rightarrow \mathrm{CO}_{2}+4 \mathrm{H}+\mathrm{C}$

Scheme S2. DCN pathways investigated in acetic acid decomposition over Pd (111).

DCN 1: $\mathrm{CH}_{3} \mathrm{COOH} \rightarrow \mathrm{CH}_{3} \mathrm{COO}+\mathrm{H} \rightarrow \mathrm{CH}_{3} \mathrm{CO}+\mathrm{H}+\mathrm{O} \rightarrow \mathrm{CO}+\mathrm{H}+\mathrm{O}+\mathrm{CH}_{3}$

DCN 2: $\mathrm{CH}_{3} \mathrm{COOH} \rightarrow \mathrm{CH}_{3} \mathrm{COO}+\mathrm{H} \rightarrow \mathrm{CH}_{2} \mathrm{COO}+2 \mathrm{H} \rightarrow \mathrm{CH}_{2} \mathrm{CO}+\mathrm{O}+2 \mathrm{H} \rightarrow \mathrm{CO}+\mathrm{O}+2 \mathrm{H}+$ $\mathrm{CH}_{2}$

$\mathrm{DCN}$ 3: $\mathrm{CH}_{3} \mathrm{COOH} \rightarrow \mathrm{CH}_{3} \mathrm{COO}+\mathrm{H} \rightarrow \mathrm{CH}_{2} \mathrm{COO}+2 \mathrm{H} \rightarrow \mathrm{CHCOO}+3 \mathrm{H} \rightarrow \mathrm{CHCO}+\mathrm{O}+3 \mathrm{H}$ $\rightarrow \mathrm{CO}+\mathrm{O}+3 \mathrm{H}+\mathrm{CH}$ 
$\mathrm{DCN} 4: \mathrm{CH}_{3} \mathrm{COOH} \rightarrow \mathrm{CH}_{3} \mathrm{COO}+\mathrm{H} \rightarrow \mathrm{CH}_{2} \mathrm{COO}+2 \mathrm{H} \rightarrow \mathrm{CHCOO}+3 \mathrm{H} \rightarrow \mathrm{CCOO}+4 \mathrm{H} \rightarrow \mathrm{CCO}$ $+4 \mathrm{H}+\mathrm{O} \rightarrow \mathrm{CO}+4 \mathrm{H}+\mathrm{O}+\mathrm{C}$

$\mathrm{DCN} 5: \mathrm{CH}_{3} \mathrm{COOH} \rightarrow \mathrm{CH}_{3} \mathrm{COO}+\mathrm{H} \rightarrow \mathrm{CH}_{3} \mathrm{CO}+\mathrm{H}+\mathrm{O} \rightarrow \mathrm{CH}_{2} \mathrm{CO}+2 \mathrm{H}+\mathrm{O} \rightarrow \mathrm{CHCO}+3 \mathrm{H}+$ $\mathrm{O} \rightarrow \mathrm{CO}+3 \mathrm{H}+\mathrm{O}+\mathrm{CH}$

DCN 6: $\mathrm{CH}_{3} \mathrm{COOH} \rightarrow \mathrm{CH}_{3} \mathrm{COO}+\mathrm{H} \rightarrow \mathrm{CH}_{2} \mathrm{COO}+2 \mathrm{H} \rightarrow \mathrm{CH}_{2} \mathrm{CO}+2 \mathrm{H}+\mathrm{O} \rightarrow \mathrm{CHCO}+3 \mathrm{H}+\mathrm{O}$ $\rightarrow \mathrm{CO}+3 \mathrm{H}+\mathrm{O}+\mathrm{CH}$

DCN 7: $\mathrm{CH}_{3} \mathrm{COOH} \rightarrow \mathrm{CH}_{2} \mathrm{COOH}+\mathrm{H} \rightarrow \mathrm{COOH}+\mathrm{H}+\mathrm{CH}_{2} \rightarrow \mathrm{CO}+\mathrm{H}+\mathrm{CH}_{2}+\mathrm{OH}$

$\mathrm{DCN}$ 8: $\mathrm{CH}_{3} \mathrm{COOH} \rightarrow \mathrm{CH}_{2} \mathrm{COOH}+\mathrm{H} \rightarrow \mathrm{CHCOOH}+2 \mathrm{H} \rightarrow \mathrm{COOH}+2 \mathrm{H}+\mathrm{CH} \rightarrow \mathrm{CO}+2 \mathrm{H}+$ $\mathrm{CH}+\mathrm{OH}$

$\mathrm{DCN}$ 9: $\mathrm{CH}_{3} \mathrm{COOH} \rightarrow \mathrm{CH}_{2} \mathrm{COOH}+\mathrm{H} \rightarrow \mathrm{CHCOOH}+2 \mathrm{H} \rightarrow \mathrm{CCOOH}+3 \mathrm{H} \rightarrow \mathrm{COOH}+3 \mathrm{H}+$ $\mathrm{C} \rightarrow \mathrm{CO}+3 \mathrm{H}+\mathrm{C}+\mathrm{OH}$

$\mathrm{DCN}$ 10: $\mathrm{CH}_{3} \mathrm{COOH} \rightarrow \mathrm{CH}_{2} \mathrm{COOH}+\mathrm{H} \rightarrow \mathrm{CHCOOH}+2 \mathrm{H} \rightarrow \mathrm{CHCOO}+3 \mathrm{H} \rightarrow \mathrm{CHCO}+3 \mathrm{H}+$ $\mathrm{O} \rightarrow \mathrm{CO}+3 \mathrm{H}+\mathrm{O}+\mathrm{CH}$

$\mathrm{DCN}$ 11: $\mathrm{CH}_{3} \mathrm{COOH} \rightarrow \mathrm{CH}_{2} \mathrm{COOH}+\mathrm{H} \rightarrow \mathrm{CH}_{2} \mathrm{COO}+2 \mathrm{H} \rightarrow \mathrm{CH}_{2} \mathrm{CO}+2 \mathrm{H}+\mathrm{O} \rightarrow \mathrm{CHCO}+3 \mathrm{H}+$ $\mathrm{O} \rightarrow \mathrm{CO}+3 \mathrm{H}+\mathrm{O}+\mathrm{CH}$

$\mathrm{DCN}$ 12: $\mathrm{CH}_{3} \mathrm{COOH} \rightarrow \mathrm{CH}_{2} \mathrm{COOH}+\mathrm{H} \rightarrow \mathrm{CH}_{2} \mathrm{CO}+\mathrm{H}+\mathrm{OH} \rightarrow \mathrm{CO}+\mathrm{H}+\mathrm{OH}+\mathrm{CH}_{2}$

$\mathrm{DCN}$ 13: $\mathrm{CH}_{3} \mathrm{COOH} \rightarrow \mathrm{CH}_{2} \mathrm{COOH}+\mathrm{H} \rightarrow \mathrm{CH}_{2} \mathrm{COO}+2 \mathrm{H} \rightarrow \mathrm{CH}_{2} \mathrm{CO}+2 \mathrm{H}+\mathrm{O} \rightarrow \mathrm{CO}+2 \mathrm{H}+\mathrm{O}$ $+\mathrm{CH}_{2}$

$\mathrm{DCN}$ 14: $\mathrm{CH}_{3} \mathrm{COOH} \rightarrow \mathrm{CH}_{2} \mathrm{COOH}+\mathrm{H} \rightarrow \mathrm{CH}_{2} \mathrm{CO}+\mathrm{H}+\mathrm{OH} \rightarrow \mathrm{CHCO}+2 \mathrm{H}+\mathrm{OH} \rightarrow \mathrm{CO}+2 \mathrm{H}$ $+\mathrm{OH}+\mathrm{CH}$

$\mathrm{DCN}$ 15: $\mathrm{CH}_{3} \mathrm{COOH} \rightarrow \mathrm{CH}_{2} \mathrm{COOH}+\mathrm{H} \rightarrow \mathrm{CHCOOH}+2 \mathrm{H} \rightarrow \mathrm{CHCO}+2 \mathrm{H}+\mathrm{OH} \rightarrow \mathrm{CO}+2 \mathrm{H}+$ $\mathrm{OH}+\mathrm{CH}$

DCN 16: $\mathrm{CH}_{3} \mathrm{COOH} \rightarrow \mathrm{CH}_{2} \mathrm{COOH}+\mathrm{H} \rightarrow \mathrm{CHCOOH}+2 \mathrm{H} \rightarrow \mathrm{CCOOH}+3 \mathrm{H} \rightarrow \mathrm{CCOO}+4 \mathrm{H}$ $\rightarrow \mathrm{CCO}+4 \mathrm{H}+\mathrm{O} \rightarrow \mathrm{CO}+4 \mathrm{H}+\mathrm{O}+\mathrm{C}$

$\mathrm{DCN}$ 17: $\mathrm{CH}_{3} \mathrm{COOH} \rightarrow \mathrm{CH}_{2} \mathrm{COOH}+\mathrm{H} \rightarrow \mathrm{CHCOOH}+2 \mathrm{H} \rightarrow \mathrm{CCOOH}+3 \mathrm{H} \rightarrow \mathrm{CCO}+3 \mathrm{H}+\mathrm{OH}$ $\rightarrow \mathrm{CO}++3 \mathrm{H}+\mathrm{OH}+\mathrm{C}$

DCN 18: $\mathrm{CH}_{3} \mathrm{COOH} \rightarrow \mathrm{CH}_{3} \mathrm{CO}+\mathrm{OH} \rightarrow \mathrm{CO}+\mathrm{OH}+\mathrm{CH}_{3}$

DCN 19: $\mathrm{CH}_{3} \mathrm{COOH} \rightarrow \mathrm{CH}_{3} \mathrm{CO}+\mathrm{OH} \rightarrow \mathrm{CH}_{2} \mathrm{CO}+\mathrm{OH}+\mathrm{H} \rightarrow \mathrm{CO}+\mathrm{OH}+\mathrm{H}+\mathrm{CH}_{2}$

DCN 20: $\mathrm{CH}_{3} \mathrm{COOH} \rightarrow \mathrm{CH}_{3} \mathrm{CO}+\mathrm{OH} \rightarrow \mathrm{CH}_{2} \mathrm{CO}+\mathrm{OH}+\mathrm{H} \rightarrow \mathrm{CHCO}+\mathrm{OH}+2 \mathrm{H} \rightarrow \mathrm{CO}+\mathrm{OH}+$ $\mathrm{CH}+2 \mathrm{H}$

DCN 21: $\mathrm{CH}_{3} \mathrm{COOH} \rightarrow \mathrm{CH}_{3} \mathrm{CO}+\mathrm{OH} \rightarrow \mathrm{CH}_{2} \mathrm{CO}+\mathrm{OH}+\mathrm{H} \rightarrow \mathrm{CHCO}+\mathrm{OH}+2 \mathrm{H} \rightarrow \mathrm{CCO}+\mathrm{OH}$ $+3 \mathrm{H} \rightarrow \mathrm{CO}+\mathrm{OH}+3 \mathrm{H}+\mathrm{C}$

DCN 22: $\mathrm{CH}_{3} \mathrm{COOH} \rightarrow \mathrm{CH}_{3}+\mathrm{COOH} \rightarrow \mathrm{CH}_{3}+\mathrm{CO}+\mathrm{OH}$ 
Table S1. The imaginary frequency of the transition state of all elementary steps investigated for the decomposition of acetic acid on $\mathrm{Pd}(111)$.

\begin{tabular}{|c|c|c|c|}
\hline steps & \multicolumn{2}{|c|}{ surface reactions } & Imaginary frequency of the Transition \\
\hline 1 & $\mathrm{CH}_{3} \mathrm{COOH}^{*}+*$ & $\mathrm{CH}_{3} *+\mathrm{COOH}^{*}$ & $417 \mathrm{icm}^{-1}$ \\
\hline 2 & $\mathrm{CH}_{3} \mathrm{COOH}^{*}+*$ & $\mathrm{CH}_{3} \mathrm{COO}^{*}+\mathrm{H}^{*}$ & $335 \mathrm{icm}^{-1}$ \\
\hline 3 & $\mathrm{CH}_{3} \mathrm{COOH}^{*}+*$ & $\mathrm{CH}_{3} \mathrm{CO}^{*}+\mathrm{OH}^{*}$ & $207 \mathrm{icm}^{-1}$ \\
\hline 4 & $\mathrm{CH}_{3} \mathrm{COOH}^{*}+*$ & $\mathrm{CH}_{2} \mathrm{COOH}^{*}+\mathrm{H}^{*}$ & $959 \mathrm{icm}^{-1}$ \\
\hline 5 & $\mathrm{CH}_{3} \mathrm{COO}^{*}+*$ & $\mathrm{CH}_{2} \mathrm{COO}^{*}+\mathrm{H}^{*}$ & $929 \mathrm{icm}^{-1}$ \\
\hline 6 & $\mathrm{CH}_{3} \mathrm{COO}^{*}+*$ & $\mathrm{CH}_{3} *+\mathrm{CO}_{2} *$ & $456 \mathrm{icm}^{-1}$ \\
\hline 7 & $\mathrm{CH}_{3} \mathrm{COO}^{*}+*$ & $\mathrm{CH}_{3} \mathrm{CO}^{*}+\mathrm{O}^{*}$ & $261 \mathrm{icm}^{-1}$ \\
\hline 8 & $\mathrm{CH}_{2} \mathrm{COOH}^{*}+*$ & $\mathrm{COOH}^{*}+\mathrm{CH}_{2} *$ & $423 \mathrm{icm}^{-1}$ \\
\hline 9 & $\mathrm{CH}_{2} \mathrm{COOH}^{*}+*$ & $\mathrm{CH}_{2} \mathrm{CO}^{*}+\mathrm{OH}^{*}$ & $239 \mathrm{icm}^{-1}$ \\
\hline 10 & $\mathrm{CH}_{2} \mathrm{COOH}^{*}+*$ & $\mathrm{CHCOOH}^{*}+\mathrm{H}^{*}$ & $779 \mathrm{icm}^{-1}$ \\
\hline 11 & $\mathrm{CH}_{2} \mathrm{COOH}^{*}+*$ & $\mathrm{CH}_{2} \mathrm{COO}^{*}+\mathrm{H}^{*}$ & $641 \mathrm{icm}^{-1}$ \\
\hline 12 & $\mathrm{CHCOOH}^{*}+*$ & $\mathrm{CHCO}^{*}+\mathrm{OH}^{*}$ & $288 \mathrm{icm}^{-1}$ \\
\hline 13 & $\mathrm{CHCOOH}^{*}+*$ & $\mathrm{CCOOH}^{*}+\mathrm{H}^{*}$ & $277 \mathrm{icm}^{-1}$ \\
\hline 14 & $\mathrm{CHCOOH}^{*}+*$ & $\mathrm{COOH}^{*}+\mathrm{CH}^{*}$ & $454 \mathrm{icm}^{-1}$ \\
\hline 15 & $\mathrm{CHCOOH}^{*}+*$ & $\mathrm{CHCOO}^{*}+\mathrm{H}^{*}$ & $365 \mathrm{icm}^{-1}$ \\
\hline 16 & $\mathrm{CH}_{2} \mathrm{COO}^{*}+*$ & $\mathrm{CH}_{2} *+\mathrm{CO}_{2} *$ & $485 \mathrm{icm}^{-1}$ \\
\hline 17 & $\mathrm{CH}_{2} \mathrm{COO}^{*}+*$ & $\mathrm{CH}_{2} \mathrm{CO}^{*}+\mathrm{O}^{*}$ & $259 \mathrm{icm}^{-1}$ \\
\hline 18 & $\mathrm{CH}_{2} \mathrm{COO}^{*}+*$ & $\mathrm{CHCOO}^{*}+\mathrm{H}^{*}$ & $802 \mathrm{icm}^{-1}$ \\
\hline 19 & $\mathrm{CHCOO}^{*}+*$ & $\mathrm{CCOO}^{*}+\mathrm{H}^{*}$ & $871 \mathrm{icm}^{-1}$ \\
\hline 20 & $\mathrm{CHCOO}^{*}+*$ & $\mathrm{CH}^{*}+\mathrm{CO}_{2} *$ & $554 \mathrm{icm}^{-1}$ \\
\hline 21 & $\mathrm{CHCOO}^{*}+*$ & $\mathrm{CHCO}^{*}+\mathrm{O}^{*}$ & $369 \mathrm{icm}^{-1}$ \\
\hline 22 & $\mathrm{CH}_{3} \mathrm{CO}^{*}+*$ & $\mathrm{CH}_{2} \mathrm{CO}^{*}+\mathrm{H}^{*}$ & $976 \mathrm{icm}^{-1}$ \\
\hline 23 & $\mathrm{CH}_{3} \mathrm{CO}^{*}+*$ & $\mathrm{CH}_{3} *+\mathrm{CO}^{*}$ & $492 \mathrm{icm}^{-1}$ \\
\hline 24 & $\mathrm{CH}_{2} \mathrm{CO}^{*}+*$ & $\mathrm{CH}_{2} *+\mathrm{CO}^{*}$ & $418 \mathrm{icm}^{-1}$ \\
\hline 25 & $\mathrm{CH}_{2} \mathrm{CO}^{*}+*$ & $\mathrm{CHCO}^{*}+\mathrm{H}^{*}$ & $801 \mathrm{icm}^{-1}$ \\
\hline 26 & $\mathrm{CHCO}^{*}+*$ & $\mathrm{CH}^{*}+\mathrm{CO}^{*}$ & $484 \mathrm{icm}^{-2}$ \\
\hline 27 & $\mathrm{CHCO}^{*}+*$ & $\mathrm{CCO}^{*}+\mathrm{H}^{*}$ & $755 \mathrm{icm}^{-2}$ \\
\hline 28 & $\mathrm{CCOOH}^{*}+*$ & $\mathrm{COOH}^{*}+\mathrm{C}^{*}$ & $492 \mathrm{icm}^{-2}$ \\
\hline 29 & $\mathrm{CCOOH}^{*}+*$ & $\mathrm{CCOO}^{*}+\mathrm{H}^{*}$ & $525 \mathrm{icm}^{-2}$ \\
\hline
\end{tabular}




\begin{tabular}{|c|c|c|c|}
\hline 30 & $\mathrm{CCOOH}^{*}+*$ & $\mathrm{CCO}^{*}+\mathrm{OH}^{*}$ & $135 \mathrm{icm}^{-2}$ \\
\hline 31 & $\mathrm{COOH}^{*}+*$ & $\mathrm{CO}^{*}+\mathrm{OH}^{*}$ & $286 \mathrm{icm}^{-2}$ \\
\hline 32 & $\mathrm{COOH}^{*}+*$ & $\mathrm{CO}_{2} *+\mathrm{H}^{*}$ & $596 \mathrm{icm}^{-1}$ \\
\hline 33 & $\mathrm{CCOO}^{*}+*$ & $\mathrm{CO}_{2} *+\mathrm{C}^{*}$ & $370 \mathrm{icm}^{-1}$ \\
\hline 34 & $\mathrm{CCOO}^{*}+*$ & $\mathrm{CCO}^{*}+\mathrm{O}^{*}$ & $377 \mathrm{icm}^{-1}$ \\
\hline 35 & $\mathrm{CCO}^{*}+*$ & $\mathrm{CO}^{*}+\mathrm{C}^{*}$ & $421 \mathrm{icm}^{-1}$ \\
\hline 36 & $\mathrm{CH}_{3} *+*$ & $\mathrm{CH}_{2}{ }^{*}+\mathrm{H}^{*}$ & $748 \mathrm{icm}^{-1}$ \\
\hline 37 & $\mathrm{CH}_{2} *+*$ & $\mathrm{CH}^{*}+\mathrm{H}^{*}$ & $501 \mathrm{icm}^{-1}$ \\
\hline 38 & $\mathrm{CH}^{*}+*$ & $\mathrm{C}^{*}+\mathrm{H}^{*}$ & $752 \mathrm{icm}^{-1}$ \\
\hline 39 & $\mathrm{OH}^{*}+\mathrm{H}^{*}$ & $\mathrm{H}_{2} \mathrm{O}^{*}+*$ & $645 \mathrm{icm}^{-1}$ \\
\hline 40 & $\mathrm{CO}^{*}+\mathrm{O}^{*}$ & $\mathrm{CO}_{2} *+*$ & $446 i \mathrm{~cm}^{-1}$ \\
\hline 41 & $\mathrm{C}^{*}+\mathrm{O}^{*}$ & $\mathrm{CO}^{*}+*$ & $350 \mathrm{icm}^{-1}$ \\
\hline 42 & $\mathrm{OH}^{*}+*$ & $\mathrm{O}^{*}+\mathrm{H}^{*}$ & $1088 \mathrm{icm}^{-1}$ \\
\hline
\end{tabular}




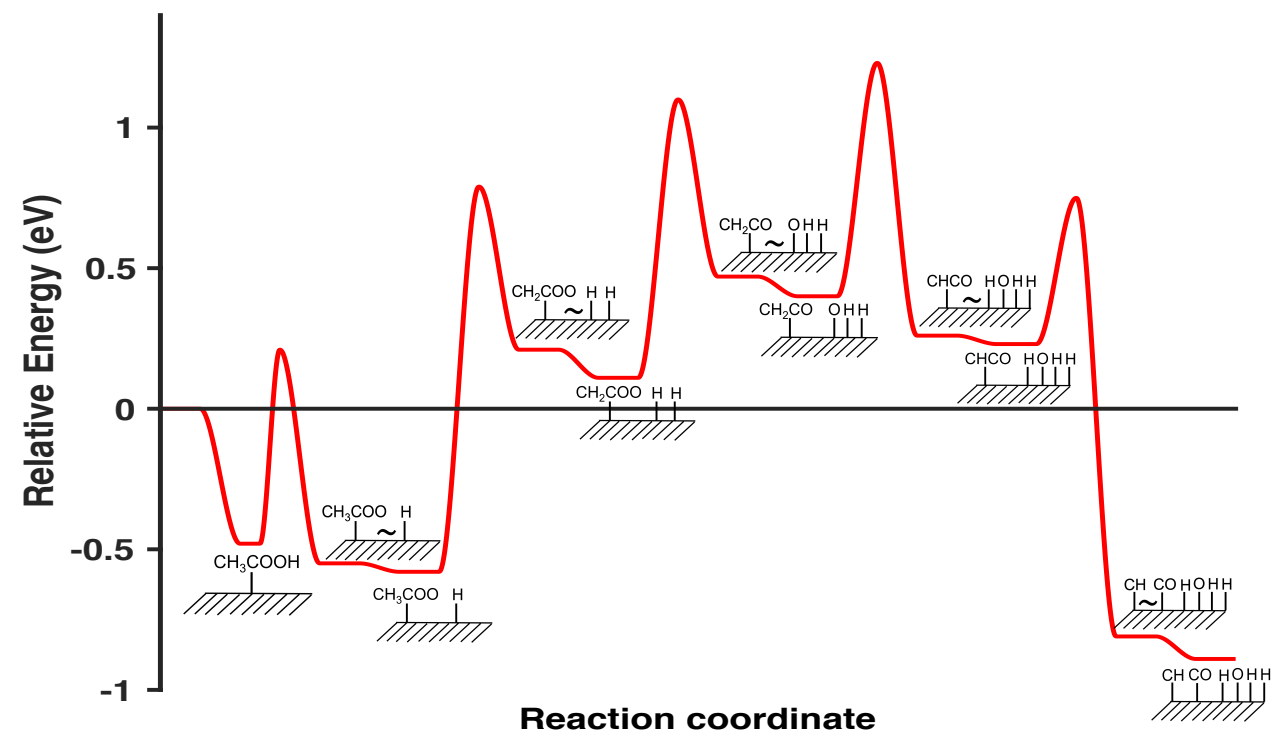

Figure S1. Energy diagram for the most favorable DCN pathways of acetic acid decomposition on Pd (111). All values shown are with respect to gas-phase acetic acid and a clean Pd (111) slab. Symbol indicates there is an interaction between the dissociated products adjacent to $\sim$. The absence of the symbol $\sim$ indicates they are infinitely separated. 


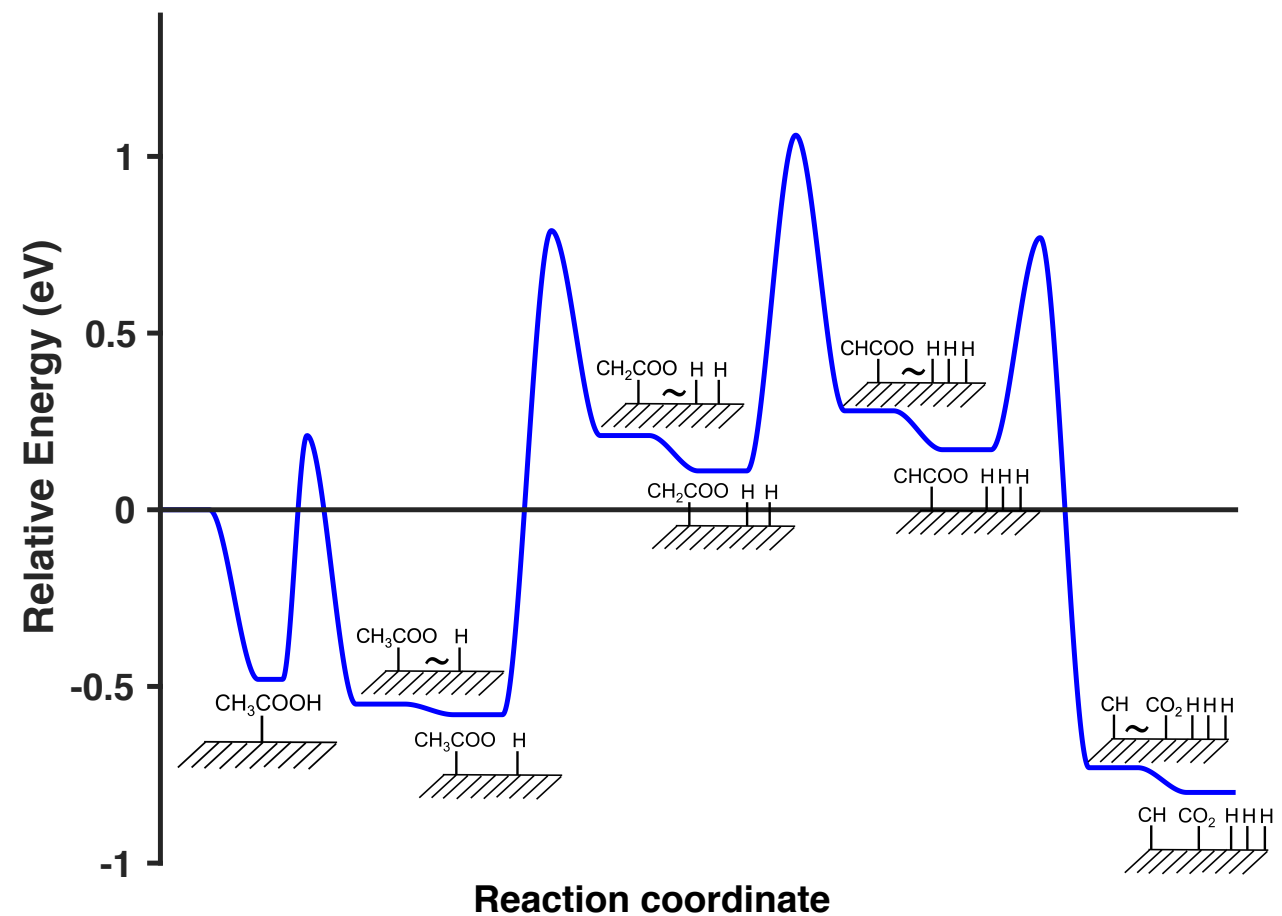

Figure S2. Energy diagram for the most favorable DCX pathways of acetic acid decomposition on Pd (111). All values shown are with respect to gas-phase acetic acid and a clean Pd (111) slab. Symbol indicates there is an interaction between the dissociated products adjacent to $\sim$. The absence of the symbol $\sim$ indicates they are infinitely separated.

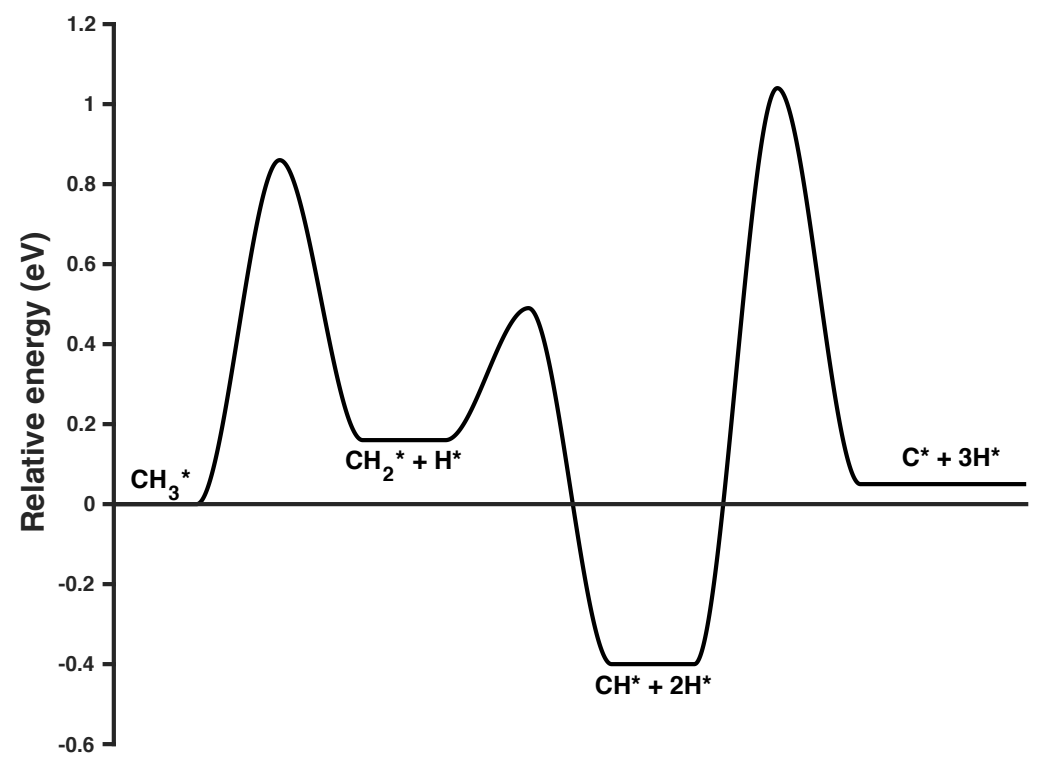

Reaction coordinate

Figure S3. Energy diagram for the $\mathrm{CH}_{3}$ decomposition to $\mathrm{C}$ and $\mathrm{H}$ on $\mathrm{Pd}(111)$. 
